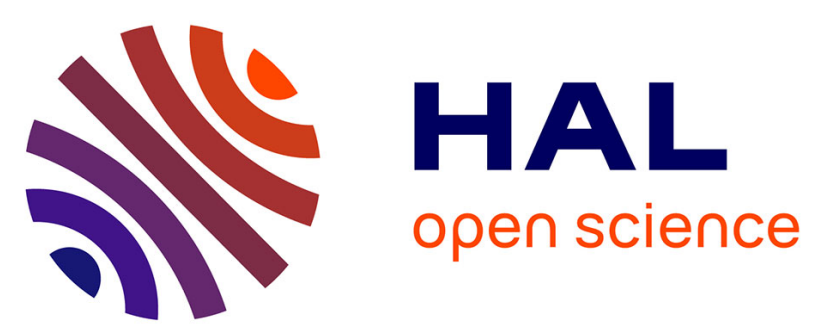

\title{
UNE ÉTUDE DU PROCESSUS DE DÉVOLUTION DES SAVOIRS EN SPORTS COLLECTIFS. ACTIVITÉ DES ÉLĖVES ET TYPE DE CONTRAT À L'ÉCOLE ÉLÉMENTAIRE (CYCLE 3)
}

Antoine Thépaut, Yvon Léziart

\section{To cite this version:}

Antoine Thépaut, Yvon Léziart. UNE ÉTUDE DU PROCESSUS DE DÉVOLUTION DES SAVOIRS EN SPORTS COLLECTIFS. ACTIVITÉ DES ÉLÈVES ET TYPE DE CONTRAT À L'ÉCOLE ÉLÉMENTAIRE (CYCLE 3). STAPS : Revue internationale des sciences du sport et de l'éducation physique, 2008, 79 (79), pp.67-80. 10.3917/sta.079.0067 . hal-03196332

\section{HAL Id: hal-03196332 \\ https://hal.univ-lille.fr/hal-03196332}

Submitted on 30 Aug 2021

HAL is a multi-disciplinary open access archive for the deposit and dissemination of scientific research documents, whether they are published or not. The documents may come from teaching and research institutions in France or abroad, or from public or private research centers.
L'archive ouverte pluridisciplinaire HAL, est destinée au dépôt et à la diffusion de documents scientifiques de niveau recherche, publiés ou non, émanant des établissements d'enseignement et de recherche français ou étrangers, des laboratoires publics ou privés. 


\section{UNE ÉTUDE DU PROCESSUS DE DÉVOLUTION DES SAVOIRS EN SPORTS COLLECTIFS. ACTIVITÉ DES ÉLĖVES ET TYPE DE CONTRAT À L'ÉCOLE ÉLÉMENTAIRE (CYCLE 3)}

Antoine Thépaut, Yvon Léziart

De Boeck Supérieur | «Staps »

2008/1 nº 79 | pages 67 à 80

ISSN 0247-106X

ISBN 9782804157777

Article disponible en ligne à l'adresse :

https://www.cairn.info/revue-staps-2008-1-page-67.htm

\section{Pour citer cet article :}

Antoine Thépaut, Yvon Léziart « Une étude du processus de dévolution des savoirs en sports collectifs. Activité des élèves et type de contrat à l'école élémentaire (cycle 3) », Staps 2008/1 (n 79), p. 67-80.

DOI 10.3917/sta.079.0067

Distribution électronique Cairn.info pour De Boeck Supérieur.

(C) De Boeck Supérieur. Tous droits réservés pour tous pays.

La reproduction ou représentation de cet article, notamment par photocopie, n'est autorisée que dans les limites des conditions générales d'utilisation du site ou, le cas échéant, des conditions générales de la licence souscrite par votre établissement. Toute autre reproduction ou représentation, en tout ou partie, sous quelque forme et de quelque manière que ce soit, est interdite sauf accord préalable et écrit de l'éditeur, en dehors des cas prévus par la législation en vigueur en France. Il est précisé que son stockage dans une base de données est également interdit. 
Antoine THÉPAUT

IUFM Nord-Pas-de-Calais

Laboratoire Théodile (E.A. 1764)

29 allée du Tardenois

59650 Villeneuve d'Ascq

antoine.thepaut@lille.iufm.fr

Yvon LÉZIART

UFR APS Université Rennes 2

Campus La Harpe

Avenue C. Tillon

35044 Rennes cedex

Laboratoire CREAD

\section{Une étude du processus de dévolution des savoirs en sports collectifs. Activité des élèves et type de contrat à l'école élémentaire (cycle 3)}

RésumÉ : Cet article étudie le processus de dévolution du savoir au cours d'un épisode d'apprentissage en basket-ball à l'école élémentaire. Il analyse l'activité des élèves dans une perspective systémique, c'està-dire dans ses relations avec les deux autres composantes du système didactique : le maître, le savoir. Les observations réalisées montrent que les élèves, confrontés à un problème posé par le maître, cherchent et expérimentent des solutions. Ces élèves sont bien en interaction avec «le milieu didactique ». Toutefois, ces solutions, faute d'être relevées, discutées et analysées avec l'enseignant, sont retenues ou non par les élèves au gré de leurs réussites ou de leurs échecs. Ceci caractérise un « contrat d'apprentissage empiriste » qui apparaît significatif d'une pédagogie de l'aménagement du milieu, modèle pédagogique fréquemment observé en EPS à ce niveau de la scolarité.

MOTS-CLÉS : dévolution des savoirs, contrat didactique, éducation physique et sportive, basket-ball, école élémentaire.

\section{AвSTRACT: A study of the process of devolution knowledge in team sports. Pupils' activity and sort of contract in elementary school}

This article studies the process of devolution of knowledge (i.e. the passing of an item or of a right from somebody to somebody else) during an episode of training in basketball at the elementary school. It analyses the activity of the pupils in a systemic perspective that is to say in its relation to the other action of the didactic process: the master and the knowledge itself.

The observations carried out show that the pupils, confronted to a problem, seek and try out solutions. They are really in interaction with the "environment". However, for want of being observed, discussed or analysed, their solutions are adopted or not by the pupils according to their successes or their failures. This characterizes an "empirical contract of apprentice ship" which indicates a form of pedagogy of "installation of the "environment", a model of teaching often observed in Physical Education at this level of schooling.

KEY WORDS: devolution of knowledge, didactic contract, Physical Education, Basketball, elementary school.

L'EPS, en tant que discipline scolaire transmet des savoirs fondamentaux et spécifiques qui participent à la formation générale et au développement harmonieux, équilibré de la personne. Si nous ne mettons pas en cause le bien-fondé d'une telle affirmation il convient cependant de s'interroger sur ce qu'apprennent réellement tous les élèves. En effet, « rappelons qu'en éducation physique et sportive les objectifs qui sont assignés à cet enseignement lui font obligation de s'adresser à tous les élèves. Aussi intéressantes que puissent être les possibilités ou aptitudes de certains, celles-ci, à aucun 
instant, ne peuvent faire oublier l'intérêt qui doit être porté à tous " (Circulaire ministère Éducation nationale, 1994). L'EPS doit permettre à tous les élèves d'apprendre. Or, si beaucoup d'élèves disent aimer cette discipline diverses enquêtes viennent régulièrement le rappeler -, en même temps, nombreux sont ceux qui disent être en difficulté, ne pas arriver à réaliser ce que l'enseignant demande et de loin en loin se construisent un sentiment d'incapacité, un sentiment d'échec.

Qu'apprennent donc les élèves ? Qu'est-ce qui, à un moment donné de l'expérience scolaire, amène un certain nombre d'entre eux à se sentir en situation d'échec, à se construire une image négative d'eux-mêmes, de la discipline et à abandonner tout espoir de progrès et de réussite?

Bien sûr, ici, de multiples facteurs interviennent, liés à la personnalité du sujet, à son histoire, à sa culture... Mais d'autres proviennent des modalités de l'enseignement proposées au cours des séances. C'est précisément ce que nous choisissons d'étudier en abordant la question de l'enseignement des jeux sportifs collectifs à l'école élémentaire. Cet enseignement est fréquemment proposé à ce niveau de la scolarité et s'appuie sur des activités physiques et sportives (les jeux sportifs collectifs) qui ont un fort retentissement affectif et émotionnel chez les élèves.

La situation de l'enseignement des jeux sportifs collectifs à l'école élémentaire en France, apparaît complexe et multiforme.

Cet enseignement est défini par des Instructions officielles formulées parfois en termes très généraux ("Au travers des pratiques qui sont les siennes [...] l'EPS, discipline d'enseignement, doit permettre à l'élève au cycle des apprentissages fondamentaux, d'acquérir des règles de l'action motrice et individuelle [...] À l'issue du cycle, l'élève doit être capable [...] d'agir en fonction des autres, selon des règles, et de tenir divers rôles dans une équipe " (B.O.E.N. $n^{\circ} 5$, 9 mars 1995), parfois en prescriptions très pré- cises définissant différents niveaux de compétences par lesquels les élèves doivent passer (Compléments aux I.O. 1984, ou B.O.E.N.

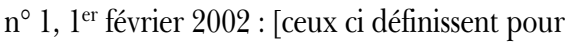
les] Jeux collectifs : se démarquer dans un espace libre, recevoir une balle, progresser vers l'avant et la passer ou tirer-marquer dans une position favorable...).

Les pratiques d'enseignement oscillent entre un «modèle traditionnel ", fondé sur les jeux de la culture enfantine et du patrimoine, organisés en séances ponctuelles sans véritable continuité, et un modèle que nous qualifions de «modèle sportif » s'appuyant sur l'enseignement des sports collectifs institutionnalisés, organisés en cycle d'apprentissage (Thépaut, 1992).

Il existe une littérature pédagogique et professionnelle abondante, traduisant cette multiplicité des pratiques, allant de fiches descriptives de jeux, jusqu'à la construction de cycles d'enseignement complets, prêts à l'emploi.

Enfin, dans la littérature plus spécialisée, il apparaît quelques recherches (Durand, 1987 ; Hay, 1988). Elles portent essentiellement sur l'apprentissage et le développement psychomoteur de l'enfant. Toutefois, si celles-ci éclairent certains aspects de l'activité des enfants, elles ne trouvent pas d'écho chez les praticiens. Ceux-ci les perçoivent très éloignées de leurs préoccupations parce qu'elles ne prennent pas en compte les conditions réelles de l'enseignement.

Cette situation ne permet pas de définir précisément ce qu'il y a à apprendre et ce qui s'enseigne effectivement en jeux sportifs collectifs à ce niveau de la scolarité. Elle rend alors difficile la réponse à la question de ce qu'apprennent réellement les élèves en EPS (tous les élèves), et en quoi les modalités d'enseignement mises en œuvre par les maitres peuvent être créatrices de réussite ou d'échec d'apprentissage chez les élèves. C'est ce que nous avons voulu rechercher, en observant précisément, au cours d'une situation réelle d'enseignement de jeu sportif collectif, si les élèves apprennent bien ce que l'enseignant souhaite leur faire acquérir. 


\section{CADRE THÉORIQUE}

Pour réaliser cette étude, nous nous appuyons sur la «théorie des situations didactiques » (Brousseau, 1986, 1998). Celle-ci nous invite à penser l'analyse du processus d'enseignement-apprentissage d'un point de vue systémique, en considérant dans une perspective d'ensemble les transformations successives du système enseignant-élève-savoir. Nous utilisons cette perspective, appréhendée comme outil d'observation et d'analyse, pour voir ce qu'elle permet de mettre en évidence à propos de l'enseignement de l'EPS.

La théorie des situations didactiques, construite pour l'étude des phénomènes d'enseignement et d'apprentissage en mathématiques a donné lieu à des extensions dans d'autres disciplines scolaires, notamment en EPS. Différentes recherches utilisant ce cadre théorique en ont alors montré la portée heuristique (AmadeEscot, 2005).

Dans le cadre de cet article, nous exploitons essentiellement le concept de la dévolution du savoir. Souhaitant étudier comment l'enseignant s'y prend pour permettre aux élèves d'apprendre et comment les élèves s'y prennent pour acquérir le savoir visé par le maître, nous avons cherché à identifier le fonctionnement du processus de dévolution d'un savoir au cours d'une séquence d'apprentissage.

G. Brousseau définit la dévolution comme «l'acte par lequel l'enseignant fait accepter à l'élève la responsabilité d'une situation d'apprentissage (a-didactique) ou d'un problème et accepte lui-même les conséquences de ce transfert » (Brousseau, 1988).

Aussi, étudier la dévolution d'un savoir au cours d'une situation d'enseignement-apprentissage, suppose donc d'identifier comment un enseignant s'y prend pour parvenir à ce que l'élève réalise volontairement, avec un taux de réussite significatif, une prestation traduisant l'acquisition du savoir en jeu et comment cet élève s'organise pour parvenir à la prestation visée, ce qu'il retient de ce que lui dit le maître, comment il interprète ce que ce dernier lui demande. Il s'agit donc d'étudier les interactions qui se nouent entre le maitre et l'élève à propos du savoir en jeu.

Dans cet article, nous abordons l'étude du processus par l'analyse du travail de l'élève. Nous entrons donc dans le système didactique - maitre-élève-savoir - par le pôle de l'activité élève, activité analysée dans son interaction avec les deux autres composantes du système.

Aussi, nous avons cherché à déterminer comment, lorsqu'un enseignant propose à ses élèves une situation d'apprentissage en jeu sportif collectif, ceux-ci construisent leur réponse, comment ils réinterprètent la situation mise en place, ce qu'ils retiennent de ce que leur dit le maître, s'ils en tiennent compte.

Pour interpréter l'activité des élèves, nous nous appuyons sur la conception piagétienne de l'apprentissage conçue comme activité adaptative du sujet face à un milieu. Analyser l'activité du sujet nécessite alors d'étudier le milieu comme ensemble organisé et système de contraintes d'une part, et le sujet confronté à ce milieu d'autre part.

«Le système antagoniste du joueur dans une situation est pour le joueur comme pour l'observateur, une modélisation de la partie de l'univers à laquelle se réfèrent la connaissance en jeu et les interactions qu'elle détermine.

C'est ce système antagoniste que nous avons proposé d'appeler milieu. Il joue donc un rôle central dans l'apprentissage, comme cause des adaptations et dans l'enseignement comme référence et objet épistémologique " (Brousseau, 1988, p. 321).

L'étude du milieu renvoie à l'analyse des jeux sportifs collectifs. Ceux-ci sont l'objet de nombreux travaux qui permettent d'en proposer des modélisations intéressantes. Deux types d'approches peuvent être retenues, l'une centrée sur l'étude des tâches motrices (Famose, 1991), l'autre sur l'analyse des jeux sportifs collectifs 
(A.E.E.P.S., 1984 ; Deleplace, 1979 ; Grehaigne, Billard \& Laroche, 1999 ; Malho, 1974 ; Mérand, 1960). Elles permettent de définir et d'étudier ces activités physiques comme un affrontement codifié entre deux équipes par l'intermédiaire d'un objet (le plus souvent un ballon), dont le but est de placer cet objet dans la cible adverse, renvoyant à une analyse du joueur dans son rapport à l'objet, la balle, son rapport avec les partenaires et les adversaires, et son rapport à l'action collective.

L'étude de l'activité du sujet renvoie à l'analyse de ses actions motrices. En nous appuyant sur la modélisation proposée par Ripoll (1985), il est possible d'avancer que l'action, organisée à partir d'une intention structurant la perception de l'environnement et les conséquences sensorielles attendues, planifie les différentes séquences motrices. Cet ensemble d'éléments se matérialise par la production d'un résultat, d'un comportement visible. L'observation et le décryptage de ce produit final permettent de reconstruire à posteriori la logique de joueur en action. Le repérage de la succession des actions permet d'identifier les différents éléments que le joueur prend en compte pour construire sa réponse et comment il s'y prend pour résoudre le problème de jeu.

Nous estimons que l'élève ne joue pas de façon inconsidérée. Lorsqu'il produit plusieurs fois de suite le même comportement, il est, selon nous, guidé par une intention élaborée à partir de ce qu'il sait déjà faire et des éléments qu'il perçoit de la situation de jeu, intention qui l'amène à produire cette réponse. C'est ici que la notion d'intention d'action apparaît pertinente. À partir de l'observation des comportements en jeu, un expert peut, en tenant compte des paramètres des tâches motrices impliquées dans un jeu sportif collectif, inférer, à partir d'un certain nombre d'hypothèses, les éléments que l'enfant, le joueur prend en compte pour produire ses réponses. Nous nous appuyons sur différents travaux fondés sur l'observation et la compréhension de l'activité de joueur (Marsenach et al., 1974 ; Marsenach, 1994).

Il est alors possible, pensons-nous, de reconstruire la cohérence de l'activité du joueur et de déterminer, pour ce qui nous concerne, comment l'élève joue dans les jeux sportifs collectifs proposés par l'enseignant ou réalise les exercices demandés.

$\mathrm{Au}$ départ, les actions, s'appuyant sur les savoir-faire initiaux, disponibles, produisent des résultats aléatoires, incertains. Lorsqu'elles se stabilisent et apparaissent avec régularité, elles permettent de penser qu'elles sont mises en œuvre de façon consciente et volontaire par le sujet. À partir de là et en reprenant à notre compte la démarche proposée par Richard (1990) pour l'étude des activités mentales nous pensons qu'il est possible de reconstruire la logique du joueur en action.

L'analyse du processus de dévolution du savoir au cours d'une situation d'apprentissage est alors l'étude du point de rencontre entre le maitre, ce qu'il sait de l'activité jeu sportif collectif et l'élève, ce qu'il retient de ses interactions avec le milieu et de ce que lui demande, lui dit le maittre. Pour l'analyse de l'activité de l'élève, il nous apparaît nécessaire de dissocier, comme le suggèrent Gréhaigne, Billard \& Laroche (1999), deux types d'activité : « ... dans l'utilisation des connaissance, quelle est l'activité du joueur en match et quelle est celle du joueur en situation d'apprentissage... L'activité cognitive du joueur n'est pas de même nature selon qu'il se situe en match avec ou sans ballon ou en situation d'apprentissage... ».

Aussi, pour inscrire les élèves sur des stratégies d'apprentissage, l'enseignant ne doit-il pas amener l'élève à distinguer nettement ce qui relève de la situation d'apprentissage de la situation de match, de telle sorte que pour l'élève, l'enjeu de la situation soit enjeu d'apprentissage et non enjeu du match ? 


\section{LE CONTEXTE DE L'ÉTUdE}

Le travail rapporté ici porte sur l'observation didactique d'une phase d'apprentissage avec une classe de CM1-CM2 (20 élèves) au cours d'un cycle de basket-ball. Elle se déroule au cours de la deuxième et de la troisième séance d'un cycle qui en comporte six.

Le maitre souhaite que les élèves apprennent à jouer en se faisant des passes. Pour cela, il aborde un premier thème d'étude qui consiste à faire comprendre aux élèves que la balle progresse plus vite lorsqu'ils se font des passes que lorsqu'ils se déplacent avec celle-ci. Pour faire progresser la balle vers l'avant, en s'appuyant sur un jeu de passes, il faut que le joueur, lorsqu'il récupère la balle, la lance à un partenaire situé en avant. Cette action suppose de la part des partenaires un déplacement rapide en avant du porteur de balle $(\mathrm{PdB})$ pour lui offrir une solution d'échange. C'est ce que le maître souhaite voir appliquer par les élèves.

L'objectif visé est donc d'apprendre aux élèves, non-porteurs de balle (NPdB), à se déplacer pour se porter en avant du PdB. La compétence recherchée porte donc sur l'action des NPdB.

Le maître propose un exercice où un problème est présenté sous une forme jouée : la balle au capitaine.

\subsection{Description du jeu}

Le but du jeu consiste à faire parvenir la balle à son capitaine, joueur situé de l'autre côté du terrain, dans une cible (cerceau).

Le dispositif est composé de 2 équipes, 1 ballon.

Les règles du jeu sont celles du basket-ball. Elles sont rappelées aux élèves à chaque début de séance.

Le maitre introduit une règle particulière, le joueur PdB n'a pas le droit de se déplacer avec la balle, quand il capte la balle, il doit s'arrêter.

\subsection{Les contenus visés}

L'enseignant souhaite que les joueurs NPdB avancent rapidement, se portent en avant du PdB.

La transformation recherchée est un jeu de coopération où les joueurs NPdB doivent se déplacer vers l'avant afin de donner au $\mathrm{PdB}$ des possibilités d'échange de la balle. Elle mobilise deux aspects de la conduite du joueur : le plan tactique, le plan de la réalisation motrice.

Au plan tactique le comportement recherché nécessite une lecture du jeu prenant en compte une vision de l'espace orienté. La cible (ici le capitaine) donne le sens du déplacement. Le savoir à mettre en œuvre consiste donc pour les NPdB, dès qu'un partenaire entre en possession de la balle, à :

- courir vers la cible,

- tout en regardant l'espace de jeu afin d'aller là où il y a ni adversaire (se démarquer), ni partenaire (écarter le jeu),

Figure 1. Représentation d'une action de jeu en balle au capitaine
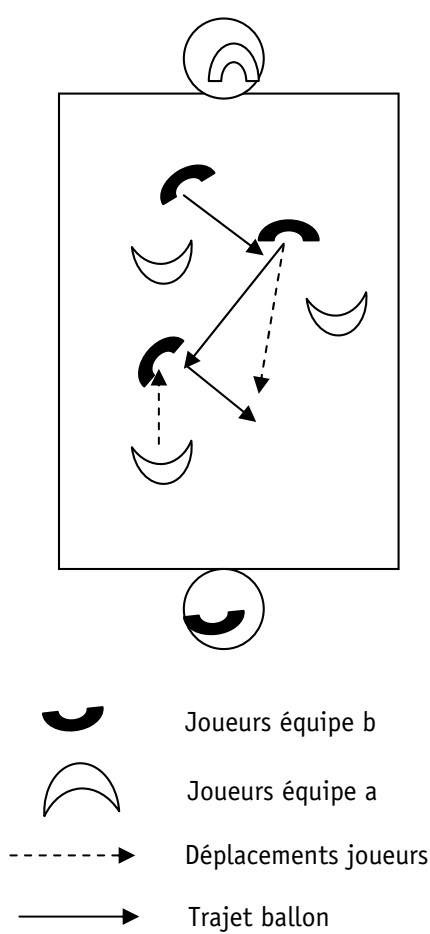
- avec une trajectoire permettant de voir simultanément la cible, le $\mathrm{PdB}$ (d'où vient la balle), la présence d'adversaires éventuels,

- en appelant la balle, c'est-à-dire en donnant des indications au PdB sur un espace fictif dans lequel le joueur NPdB, futur réceptionneur, est susceptible de pouvoir capter la balle (Falguières et Muguet, 1990).

- Au plan de la réalisation motrice le comportement recherché nécessite un ajustement entre la trajectoire de la balle et la trajectoire de son propre déplacement. Il s'agit d'entrer en coïncidence avec la trajectoire de la balle, ce qui suppose une lecture de cette trajectoire afin d'estimer le point de chute de la balle, tout en allant à la rencontre de la balle pour limiter les risques d'interception par l'adversaire et avec une orientation corporelle «oblique " permettant d'avoir dans le même champ visuel le PdB transmetteur et la cible à atteindre.

\subsection{Un épisode}

Cet exercice est présenté dans des conditions de jeu à effectifs réduits $(5 \times 5)$, sous la forme d'atelier. Situé entre l'échauffement et une phase de jeu global, il a le statut de situation d'apprentissage, situation didactique (Brousseau, 1986). Pendant qu'une moitié de l'effectif classe travaille à un exercice de " passe et tir au panier ", l'autre moitié s'exerce à cet atelier de la «balle au capitaine ». L'enseignant considère cet atelier comme l'atelier principal, il y reste en permanence.

Au bout de cinq minutes, l'enseignant procède à une rotation des groupes.

Nous avons ainsi deux séquences de cinq minutes chacune au cours de la deuxième séance. Comme cette première série ne donne pas les résultats escomptés, l'enseignant la prolonge au cours de la troisième séance.

Tableau 1. Structuration temporelle des séances observées

\begin{tabular}{|c|c|c|c|c|}
\hline & \multicolumn{2}{|c|}{ Séance 2} & \multicolumn{2}{|c|}{ Séance 3} \\
\hline & \multicolumn{2}{|c|}{ Échauffement } & \multicolumn{2}{|c|}{ Échauffement } \\
\hline \multirow{2}{*}{$\begin{array}{l}\text { Exercice de la balle au } \\
\text { capitaine } \\
\text { (4 séquences) }\end{array}$} & $\begin{array}{l}\text { Séquence } 1 \\
1 / 2 \text { Groupe a }\end{array}$ & $\begin{array}{l}\text { Atelier tir au panier } \\
1 / 2 \text { Groupe b }\end{array}$ & $\begin{array}{l}\text { Séquence } 3 \\
1 / 2 \text { Groupe a' }\end{array}$ & $\begin{array}{c}\text { Atelier tir au panier } \\
\text { 1/2 Groupe b' }\end{array}$ \\
\hline & $\begin{array}{l}\text { Atelier tir au panier } \\
\text { 1/2 Groupe a }\end{array}$ & $\begin{array}{l}\text { Séquence } 2 \\
1 \text { ×2 Groupe b }\end{array}$ & $\begin{array}{l}\text { Atelier tir au panier } \\
\text { 1/2 Groupe a' }\end{array}$ & $\begin{array}{l}\text { Séquence } 4 \\
\text { 1⁄2 Groupe b' }\end{array}$ \\
\hline $\begin{array}{l}\text { Séquence de matchs } \\
\text { de fin de séance }\end{array}$ & \multicolumn{2}{|c|}{$\begin{array}{l}\text { Match } 1 \\
\text { Match } 2\end{array}$} & \multicolumn{2}{|c|}{$\begin{array}{l}\text { Match } 3 \\
\text { Match } 4\end{array}$} \\
\hline
\end{tabular}

Source : N.B. : Les 1 ² groupes sont dénommés a et a', b et b' car ils ne sont pas constitués avec les mêmes élèves d'une séance sur l'autre.

Quatre séquences d'environ cinq minutes chacune sont donc consacrées à cet apprentissage. Elles constituent, selon la classification établie par Amade-Escot (1998), un même épisode.

\section{MÉTHODE}

\subsection{Le recueil des données}

Chacune des séquences est filmée à l'aide de deux caméras, permettant de couvrir tout l'espace de jeu. Les enregistrements donnent lieu à une transcription du discours et des actions de l'enseignant en même temps qu'un relevé de l'activité des élèves.

Procédant à une réduction des données, nous n'avons retenu des actions et discours de l'enseignant que les interventions d'ordre didactique, c'est-à-dire celles ayant trait spécifiquement à la communication des savoirs (AmadeEscot et Marsenach, 1995 ; Sensevy, Mercier \& Schubauer-Léoni, 2000). Nous extrayons du discours de l'enseignant, à l'image de ce qui se fait dans les études en didactique des mathématiques, toutes les interventions " qui ne contiennent pas d'énoncés mathématiques comme ceux 
qui organisent et contrôlent les actions des élèves dans la classe afin que les activités strictement mathématiques puissent avoir lieu» (Nadot, 1997). Nous obtenons alors ce que l'auteur appelle un « discours réduit ». Nous réalisons pour cela une analyse de contenu à l'aide de la catégorisation proposée par Marsenach \& Mérand (1987).

Pour ce qui concerne l'activité des élèves, nous n'avons retenu que leurs déplacements en tant que NPdB. Ainsi, à chaque échange de la balle, à chaque passe, nous relevons le déplacement (ou non déplacement) des partenaires du porteur de balle.

Nous établissons ensuite une "chronique " des séquences étudiées (Leutenegger, 2000) mettant en parallèle les interventions verbales d'ordre didactique de l'enseignant et les stratégies de résolution du problème tentées par les élèves.

\subsection{Analyse des données}

Pour analyser l'activité des élèves, nous avons repris pour chacun d'eux, la succession des actions de déplacement en tant que NPdB. À chaque fois qu'un joueur entre en possession de la balle, pour chacun de ses partenaires, nous nous demandons si ceux-ci se déplacent ou non, si oui où, en restant en arrière $\mathrm{du} \mathrm{PdB}$, en avant, avec quelle orientation ? Nous avons alors cherché à travers l'enchaînement de leurs essais, si des régularités apparaissent dans la succession de leurs actions, et à partir de là, à identifier par inférence les paramètres qu'ils prennent en compte pour construire leur réponse.

Ainsi, le maître souhaite que les élèves apprennent, lorsqu'ils sont NPdB, à se déplacer en avant du PdB. Pour cela, il construit un «milieu didactique » (Brousseau, 1988) posant un problème de jeu à résoudre. Nous recherchons, à partir de l'observation et de l'analyse de l'activité de chacun des élèves, si ceux-ci par- viennent à résoudre ce problème, du moins s'ils cherchent des solutions pour réussir, et en cela, s'ils assument la responsabilité de la situation d'apprentissage.

\section{RÉSULTATS}

\subsection{L'activité des élèves}

Le relevé des modes de réalisation de l'exercice permet, pour chaque élève, une comparaison entre les stratégies utilisées d'une séquence à l'autre. Nous observons ${ }^{1}$ :

- Neuf élèves ne changent pas de stratégie d'une séquence à l'autre :

- Trois éludent le problème: soit ils ne se déplacent pas, évitant ainsi de se placer en position de recevoir la balle, soit ils profitent de la première occasion pour «sortir du jeu» en prenant la place de capitaine.

- Deux sont en difficulté et ne parviennent pas à résoudre le problème. Malgré leur implication effective en jeu, leurs difficultés motrices (difficultés de déplacement et de capture de la balle), leur état fusionnel avec la balle les amènent à suivre les déplacements du ballon et non à s'organiser pour être en position de le recevoir.

- Quatre ont un comportement stabilisé qu'ils reproduisent d'une séquence à l'autre. Il s'agit d'une solution qui, dans le problème posé, est une solution efficace, amenant une réussite certaine. Dans ces conditions, ces élèves n'estiment pas nécessaire de changer de stratégie. Ils ne sont pas dans des conditions de jeu nécessitant un changement de stratégie.

- 4 élèves changent de stratégie d'une séquence à l'autre

- 4 élèves changent de stratégie au cours d'une même séquence

1. Seuls 17 élèves ont pu être observés au cours des 2 séquences. Les prises de vues ont été réalisées au cours du mois de mars, période de l'année où la classe comptait plusieurs élèves malades, les uns étant absents à la $2^{\mathrm{e}}$ séance, les autres à la $3^{\mathrm{e}}$. 


\subsection{Des changements de stratégie}

Plusieurs facteurs sont à l'origine des changements de stratégie.

\subsection{1. des injonctions de l'enseignant après une action de l'élève}

- Ayant entraîné la réussite : un joueur reçoit la balle, ses partenaires n'avancent pas. Le mầtre intervient verbalement "avance ». L'élève court, offre une solution d'échange, reçoit la balle qu'il capte avant de relancer de nouveau (Laura Mohamed). Ici, dans le cours du jeu, lorsque le PdB reçoit la balle, ses partenaires n'avancent pas. C'est, pour ces joueurs, leur réponse initiale, sans s'apercevoir que leur absence de déplacement ne permet pas de faire progresser la balle et donc de résoudre le problème. L'enseignant leur dicte la solution à mettre en œuvre, solution qu'ils appliquent alors et qui aboutit à la réussite de l'action : ils se déplacent, offrent une opportunité reçoivent et captent la balle. Il apparait que l'injonction provoque un déclic, l'émergence de la solution qui, répétée sous la conduite de l'enseignant, est ensuite appliquée de façon autonome par l'élève.

- Sans rapport avec la réussite : une élève entre en possession de la balle. Gwendoline reste en défense près du capitaine adverse. Le maître lui commande d'avancer. Gwendoline court alors vers l'avant, sans se soucier du ballon, et part se placer loin devant, près de son capitaine.

Dans ce cas présent, l'enseignant constatant une situation de blocage, dicte à l'élève la solution à appliquer. Toutefois, l'élève interprète la commande par un déplacement sur toute la longueur du terrain. La question de l'avancée de la balle en passes successives, et donc de l'avancée des NPdB à distance raisonnable par rapport au PdB n'est pas perçue par cette élève.

L'injonction, dans la brièveté de son contenu - il faut faire vite, donner l'indication sur l'instant présent, avant qu'une nouvelle action ne vienne modifier la configuration de jeu donne la réponse à apporter, sans en préciser le sens. Elle ne fournit pas les éléments de compréhension du jeu, la nécessité de la progression de la balle et d'aide au PdB par un placement/déplacement approprié.

\subsection{2. des changements d'équipe}

Une répartition implicite des rôles s'opère au sein des équipes. Or cette distribution des rôles et le placement géographique sur le terrain qui en résulte, conditionnent les réponses apportées. Un joueur qui se place en tant que récupérateur, distributeur de balle, en arrière de l'espace de jeu effectif (E.J.E.) (Grehaigne, Billard \& Laroche, 1999) sera plus vite confronté au problème posé par l'enseignant qu'un élève qui se place systématiquement en avant de cet E.J.E.

À ce niveau, nous observons que certains joueurs, lorsqu'ils changent d'équipe entre leur première et leur seconde séquence, changent de rôle et donc de réponse.

Hafida : Au cours de la première séquence, elle reste toujours en arrière du $\mathrm{PdB}$, tout en le suivant et en appelant la balle. Lors de la deuxième séquence, personne n'occupant le poste, elle adopte la stratégie de l'avant piquet, stratégie consistant à se placer près de son capitaine (cible) et attendre qu'un partenaire lui fasse une longue passe, n'ayant plus alors qu'à donner la balle à son capitaine, sans crainte d'une interception adverse.

Anaïs : À la première séquence, elle reste en défense près du capitaine adverse, personne dans son équipe ne voulant tenir ce rôle. Lors de la deuxième séquence, elle occupe un poste de relais entre le réceptionneur distributeur et le joueur avancé de son équipe.

Laura : Durant la première séquence, elle se place toujours en avant du PdB. Au cours de la deuxième séquence, elle se place en arrière à la récupération, distribution du jeu. 
Tableau 2. Exemple de recueil de données : une élève n'applique pas la solution (Thépaut, 2002)

\begin{tabular}{|c|c|}
\hline OBSERVATIONS & COMMENTAIRES \\
\hline $\begin{array}{l}4^{\circ} \text { action } \\
\text { Gwendoline récupère la balle. } \\
\text { Elle la passe à Emeline, devant elle à } 2 \text { mètres environ. } \\
\text { Emeline se retourne. Rémi est parti vers l'avant, trop loin. Elle ne } \\
\text { peut pas lui faire la passe. } \\
\text { Elle se retourne alors et passe à Gwendoline qui n'a pas bougé. } \\
\text { Elle relance la balle à Emeline et reste sur place. Emeline se } \\
\text { retrouve dans le même cas de figure que précédemment. } \\
\text { L'enseignant commande alors à Gwendoline d'avancer : «Allez, } \\
\text { vers lavant, allez, avance. » } \\
\text { Gwendoline court, se dirige vers Rémi et se place en soutien. } \\
\text { Emeline effectue une longue passe à Rémi vers l'avant et le coté, } \\
\text { puis court vers l'avant. Gwendoline reste derrière Rémi. } \\
\text { Le maitre commande une nouvelle fois à Gwendoline d'aller vers } \\
\text { l'avant. «Allez, allez, ... va vers l'avant, allez, allez. » } \\
6 \text { action : } \\
\text { Dès que l'équipe adverse est entrée en possession du ballon, } \\
\text { Gwendoline est retournée en défense près du capitaine adverse. } \\
\text { Aussi, lorsque Rémi intercepte une passe adverse, il est obligé } \\
\text { d'attendre qu'Emeline se démarque devant, seule solution possi- } \\
\text { ble, Gwendoline est restée en défense. } \\
\text { Le maitre commande à nouveau à Gwendoline d'avancer : } \\
\text { "Gzwendoline, va l'aider un peu... allez, bouge, voilà bouge... » } \\
\text { Gwendoline court vite se placer près de son capitaine sans même } \\
\text { regarder ses partenaires et adversaires. }\end{array}$ & $\begin{array}{l}\text { Gwendoline dès le départ du jeu s'est placée en défense près du } \\
\text { capitaine adverse et y reste. } \\
\text { Aussi, lorsque Emeline entre en possession de la balle pour la } \\
\text { seconde fois et qu'elle n'a pas de solution d'échange, Rémi étant } \\
\text { trop avancé, le maître commande à Gwendoline d'avancer. } \\
\text { C'est alors ce qu'elle fait, en courant, mais pour aller se placer en } \\
\text { arrière de Rémi. Aussi, lorsque ce dernier entre en possession de } \\
\text { la balle il n'a pas de solution de passe vers l'avant. C'est ce qui } \\
\text { explique la seconde intervention de l'enseignant. Il commande à } \\
\text { nouveau à Gwendoline d'avancer. } \\
\text { C'est ce qu'elle fait alors mais pour aller se placer juste devant son } \\
\text { capitaine. }\end{array}$ \\
\hline
\end{tabular}

\subsubsection{Une opportunité de jeu : une répartition géographique des joueurs sur le terrain amène à tenter un autre comportement, avant de revenir à un comportement antérieur}

Thiffaine, lors de la première séquence, au début du jeu, elle, est en arrière du PdB. À l'issue d'une modification de la répartition des joueurs, le joueur systématiquement placé devant descend quelque peu afin de soutenir ses partenaires en défense. Thiffaine profite de cette occasion pour se placer en avant du PdB pendant 3 actions, avant de revenir à son comportement initial.

Abdelbassat, au milieu de la première séquence, constatant que son équipe ne parvient pas à faire progresser la balle, effectue une tentative de déplacement en une-deux, face à face, avec Kewin (deux actions identiques consécutives). Celles-ci se soldant par des échecs, il abandonne cette solution et revient à son 
comportement antérieur, en arrière du $\mathrm{PdB}$. Au cours de la deuxième séquence, il tente de nouveau de se déplacer en une-deux, face à face, avec Kewin. Ces deux tentatives échouent également.

\section{ANALYSE}

Au cours de ces séquences de la balle au capitaine, les élèves sont bien en interaction avec «le milieu didactique» (Brousseau, 1988) mis en place par l'enseignant. Face au problème posé par celui-ci, ils tentent de le résoudre, ils essaient des solutions, cherchent une voie, reviennent à leur première solution, essaient autre chose. Ils ne sont pas inactifs face au problème à résoudre. Toutefois, ces solutions, faute d'être observées, relevées, montrées, discutées et analysées sont retenues ou non par les élèves, au gré de leurs réussites ou de leurs échecs.

En effet, le relevé précis de l'activité et du discours de l'enseignant montre que celui-ci relève à la fin de trois séquences sur les quatre, une action observée au cours du jeu. Dans un cas, il s'agit d'une action réussie, pour montrer ce qu'il faut faire, ce qu'il attend des élèves. L'équipe vient de faire parvenir la balle à son capitaine en trois passes rapides depuis la remise en jeu. Toutefois, il insiste ici sur la brièveté de la progression et non sur le déplacement des NPdB qui, en la circonstance, étaient déjà « étagés » en trois niveaux - le récupérateur, un joueur relais, un avant-piquet - et n'ont donc pas eu à se déplacer. Dans les deux autres cas, il pointe une action qui a échoué, ce qui lui permet d'expliquer les causes de l'échec et en contrepoint, ce qu'il faut faire pour réussir. Lors de la quatrième séquence, il reprend un point, sans rapport avec le comportement attendu, qui lui permet d'introduire l'exercice suivant.

Lors de ses explications, le maître prend appui sur une action, celle d'un joueur, prise de façon ponctuelle et aléatoire.
Mais, lorsque Abdelbassat, par exemple, adopte un comportement, puis au cours de la séquence change pour adopter une solution qui s'avère prometteuse dans la transformation recherchée par le maitre, mais qui est marquée par l'échec dans son résultat (essai tenté deux fois consécutivement au cours de la première séquence, puis recommencé à l'identique, à nouveau deux fois avec le même partenaire au cours de la seconde séquence, avec toujours le même résultat, le même échec), personne ne relève ces tentatives, ne mentionne les voies prometteuses expérimentées ici. Abdelbassat aura vécu deux séquences sans que cela ne lui ait servi puisqu'il revient au final à sa première forme de réponse.

La forme de travail, les modalités adoptées par l'enseignant ne permettent pas de relever les réponses des élèves. Les solutions expérimentées, qui apparaissent à l'observation minutieuse et outillée, sont en quelque sorte laissées en suspens. Les élèves apparaissent seuls face à leur propre cheminement d'apprentissage.

Nous le voyons également, certains enfants ne changent pas de stratégie, soit parce qu'ils éludent le problème (les élèves peu investis qui profitent de la première occasion pour sortir du jeu), soit parce qu'ils sont en difficulté, soit enfin parce qu'ils ont un comportement stabilisé.

Quels éléments permettent de comprendre l'absence de prise en charge de la réponse par les élèves? Les difficultés proviennent selon nous du contrat didactique développé au cours de cet épisode, contrat didactique que nous pouvons qualifier de « contrat d'apprentissage empiriste... », où « la connaissance est supposée s'établir essentiellement par le contact avec le milieu auquel l'élève doit s'adapter. La responsabilité de l'apprentissage est renvoyée au milieu et à la nature » (Brousseau, 1996).

Ce contrat d'apprentissage empirique permet de comprendre l'origine des dysfonctionnements de contrat ainsi que les difficultés d'ap- 
prentissage rencontrées par les élèves. Les élèves sont livrés à eux-mêmes dans le choix d'une réponse au problème et dans la stabilisation de celle-ci.

\section{DISCUSSION}

Cette étude montre l'intérêt que peut avoir la prise en compte du concept de dévolution pour l'observation des phénomènes d'enseignement et d'apprentissage en EPS.

Elle montre également la portée heuristique de la notion de contrat didactique qui lui est associée. La caractérisation du type de contrat à l'œuvre au cours de cet épisode permet de repérer et de comprendre les difficultés d'apprentissage auxquelles sont confrontés les élèves. Ces difficultés ne sont pas tant liées au problème posé qu'à l'action d'accompagnement du maître. Cela ouvre bien des perspectives sur les nécessités d'analyse de l'activité du maitre en EPS. Ici, il est alors possible d'envisager d'exploiter plus complètement les travaux de G. Broussseau (1996). En effet, pour l'observation des situations d'enseignement en mathématiques, l'auteur établit une classification selon qu'elles reposent sur des contrats non didactiques, faiblement didactiques à des contrats fortement didactiques ou des contrats basés sur la transformation de savoirs «anciens ». La reprise de cette classification en EPS devrait permettre de caractériser davantage la discipline et de repérer les effets que ces différents types de contrat peuvent produire.

Pour ce qui concerne la question de l'enseignement des jeux sportifs collectifs, il existe différentes approches. Au cours d'une revue de question, Grehaigne, Godbout \& Mahut (1999) distinguent trois démarches d'enseignement, une pédagogie des modèles d'exécution, reposant sur l'apprentissage par le joueur de solutions efficaces produites par les experts, une pédagogie des modèles auto-adaptatifs s'appuyant sur des variations judicieuses d'aménagement du milieu pour faire découvrir les solutions et développer les habiletés visées, et enfin une pédagogie des modèles de décisions tactiques centrée sur la présentation des informations essentielles à l'orientation des actions en jeu (Bouthier, 1984). Or, si, comme le soulignent les auteurs, "la difficulté à mener la preuve de la supériorité de telle ou telle approche dans le domaine des apprentissages moteurs constitue un fait incontournable" (Grehaigne, Godbout \& Mahut 1999), l'étude que nous avons réalisée ici montre les limites d'une pédagogie des modèles auto-adaptatifs, autrement caractérisée comme une pédagogie de l'aménagement du milieu.

En effet, nous le voyons, l'enseignant propose une situation qui, pour être suffisamment bien agencée, suffisamment bien construite doit provoquer par la seule mise en activité des élèves, la découverte de la solution juste, de la solution efficace et par là même leur permettre d'acquérir l'habileté souhaitée. Or il n'en est rien, les réponses sont diverses, les solutions apportées multiples. Si les élèves sont bien dans une activité exploratoire, faute d'être aidés, guidés dans le processus d'apprentissage, ils ne parviennent pas à extraire de leur activité la solution efficace

Cette étude met enfin en évidence la façon dont les élèves s'engagent dans des procédures d'apprentissage spontanées au cours desquelles ils entrent peu en interaction avec le maitre. À la différence de ce que l'observation d'activités mathématiques montre (Brousseau, 1980 ; Blanchard-Laville, 1997), les élèves ne passent pas en éducation physique par une activité de décodage des intentions du maître. Il semble que l'enjeu du jeu n'est pas enjeu d'apprentissage mais enjeu en soi. Est-ce un trait spécifique à la discipline EPS, ou une caractéristique liée au fait que nous sommes en présence d'un contrat d'apprentissage empiriste ? Une multiplication du nombre d'observations de séquences portant sur l'étude du fonctionnement du contrat didactique aidera à répondre à cette question. 


\section{CONCLUSION}

L'étude du processus de dévolution du savoir à travers cet exemple, pris au cours d'un cycle d'enseignement en basket-ball à l'école élémentaire, nous montre l'enseignant construire une situation d'enseignement. L'agencement judicieux du milieu - notamment la proposition de la règle interdisant au $\mathrm{PdB}$ de se déplacer - est supposé élaborer un problème et permettre aux élèves de découvrir la solution et par là d'acquérir l'habileté visée.

Nous l'avons vu, les élèves cherchent bien à résoudre le problème qui leur est posé. La plupart essaient des solutions, changent de stratégie...

Toutefois, ces solutions expérimentées, faute d'être relevées, analysées, évaluées sont adoptées ou abandonnées par les élèves, au gré de leurs réussites et de leurs échecs.

Nous voyons là les effets d'un contrat didactique reposant sur un « contrat d'apprentissage empiriste ». Les difficultés d'apprentissage rencontrées par les élèves sont importantes. Ceuxci doivent en effet trouver par eux-mêmes la solution la plus efficace au problème posé sans garantie par le maitre de la pertinence de leur choix.

C'est ici toute la difficulté de la gestion du processus d'apprentissage de l'élève par l'enseignant. L'apprentissage moteur, nous dit Famose (1991), suppose la mise en œuvre d'une pédagogie de résolution de problème sollicitant de la part de l'élève, une activité consciente et réfléchie d'identification des règles et principes d'action ou des schémas tactiques. "La solution au problème moteur est essentiellement de nature consciente » (Famose, 1991, 37).

Or, si dans le cadre de l'enseignement des mathématiques, une telle démarche pédagogique a pu être formalisée (Brousseau, 1986), elle rencontre par contre en EPS de nombreuses résistances. Sans doute, celles-ci sont en partie liées aux dérives de la verbalisation provoquées par certaines formes de la pédagogie de l'éveil ou pédagogie de la découverte mises en œuvre dans les années 1970-1980. Les élèves passaient alors davantage de temps à écrire, dessiner ou verbaliser qu'à agir. Néanmoins, ces modèles pédagogiques cherchaient à dépasser les limites et difficultés d'un apprentissage spontanéiste en EPS. Tout n'est pas à rejeter dans ces tentatives de dépassement que représentent ces innovations pédagogiques.

D'autres expérimentations pédagogiques ont été engagées depuis, visant à permettre aux élèves de s'engager dans une activité d'identification des principes d'action tout en s'appuyant sur une activité effective et conséquente des élèves.

Cette étude de cas révèle un phénomène particulier. S'agit-il d'un fonctionnement propre à l'EPS permettant de saisir pourquoi les élèves «sont d'éternels débutants ", ou de la prégnance dans cette discipline de la pédagogie par «aménagement du milieu », ou bien encore d'un fait lié à la situation particulière d'enseignement analysée ici ? En clair, cet exemple est-il caractéristique du fonctionnement classique de l'EPS, révélant un mécanisme de fond dans les procédures d'enseignement et d'apprentissage à l'œuvre dans cette discipline ou n'est-il qu'un exemple singulier et grossissant d'une partie seulement des démarches d'enseignement proposée ?

Il conviendrait de réaliser maintenant d'autres observations, d'autres études sur ce principe. L'augmentation du nombre de séquences observées, la multiplication du nombre de données empiriques retenues et l'étude précise de ce qui se passe au cours des séances, faciliteront la compréhension des mécanismes mis en jeu par le maitre et les élèves pour que ceux-ci apprennent. 


\section{BIBLIOGRAPHIE}

A.E.E.P.S. (1984). Sports Co en milieu scolaire. Des stages amicale au nouveau bac. Paris : A.E.E.P.S.

Amade-Escot, C., Marsenach, J. (1995). Didactique de l'éducation physique et sportive. Grenoble : La Pensée Sauvage.

AmAde-Escot, C. (1998). Apport des recherches didactiques à l'analyse de l'enseignement : une étude de cas, le contrat didactique, Recherches en EPS : bilan et perspectives. Paris : Éditions Revue E.P.S.

Amade-Escot, C. (2005). Milieu, dévolution, contrat, regard de l'éducation physique, in M.H. Salin., P. Clanché, B. Sarrazy. (Éds), Sur la théorie des situations didactiques, Hommage à G. Brousseau. Grenoble : La Pensée Sauvage, 91-98.

BlanChard-Laville, C. (1997). Variations sur une leçon de mathématiques. Analyses d'une séquence : "L'écriture des grands nombres». Paris : L'Harmattan.

BOUTHIER, D. (1984). Sports collectifs : contribution à l'analyse de l'activité et éléments pour une formation tactique essentielle. L'exemple du rugby, Mémoire INSEP, Paris.

Brousseau, G. (1980). Les échecs électifs dans l'enseignement des mathématiques à l'école élémentaires. Revue de laryngologie, otologie, rhinologie, vol. 101, 3-4, 107-131.

Brousseau, G. (1986). Fondements et méthode de la didactique des mathématiques. Recherches en Didactique des Mathématiques. Grenoble: La Pensée Sauvage, vol. $7, \mathrm{n}^{\circ} 2,33-115$.

Brousseau, G. (1988). Le contrat didactique : le milieu. Recherches en Didactique des Mathématiques. Grenoble : La Pensée Sauvage, vol. 9, n³, 309-336.

Brousseau, G. (1996). L'enseignant dans la théorie des situations didactiques, Actes de la $8^{\mathrm{e}}$ école d'été de didactique des mathématiques, Noirfalise \& M.J. PerrinGlorian (Éds), Clermont-Ferrand, I.R.E.M., 3-46.

Brousseau, G. (1998). Théorie des situations didactiques, didactique des mathématiques 1970-1990. Grenoble : La Pensée Sauvage.

DelePlace, R. (1979). Rugby de mouvement - rugby total. Paris : Éditions Revue EP.S.

Falguière, C. Muguet, J.-P. (1990). Le basket-ball. Éducation Physique et Didactique des A.P.S. A.E.E.P.S., 38-49.

Durand, M. (1987). L'enfant et le sport. Paris : PUF.

FAMOSE, J.-P. (1991). Apprentissage moteur et difficulté de la tâche. Paris : INSEP.

Grehaigne, J.-F., Billard, M. \& Laroche, J.Y. (1999). L'enseignement des sports collectifs à l'école. Conception, construction et évaluation. Bruxelles : De Boeck.
Grehaigne, J.-F., Godbout, P. \& Mahut, N. (1999). L'enseignement des jeux par la compréhension : une revue de question, Revue STAPS, 48, 81 - 93.

HaY, L. (1988). Le développement du contrôle moteur. L'exemple d'un geste d'approche manuel. In M. Laurent et P. Therme (Éds) L'enfant par son corps. Paris : Actio.

Leutenegger, F. (2000). Construction d'une « clinique» pour le didactique. Une étude des phénomènes temporels de l'enseignement. Recherches en Didactique des Mathématiques. 20, 2, 209-250.

MaLHo, F. (1974). L'acte tactique en jeu. Paris : Vigot.

Marsenach, J., Druenne, F. (1974). Volley-ball. Mémento CPS FSGT. Paris : Sport et Plein-Air.

Marsenach, J., MÉrand, R. (1987). L'évaluation formative en EPS dans les collèges. Paris : INRP, Rapport de recherche $n^{\circ} 2$.

Marsenach, J. (1994). EPS au collège et volley-ball. Paris : INRP.

MÉrand, R. (1960). Le basket-ball, jeu simple, Revue EPS, $n^{\circ} 50,51-53$.

Ministère Éducation Nationale (1984). Compléments aux instructions officielles.

Ministère Éducation Nationale (1994). Épreuves au baccalauréat, B.O.E.N., n ${ }^{\circ}$, Circulaire $n^{\circ} 94-007$.

Ministère Éducation Nationale (1995). B.O.E.N., n $n^{\circ} 5$ du 9 mars.

Ministère Éducation Nationale (2002). Programmes de l'école primaire. Cycle des approfondissements. B.O.E.N., $\mathrm{n}^{\circ} 1 \mathrm{du} 1^{\circ}$ février.

Nadot, S. (1997). Obstacles et apprentissages, in C. Blanchard-Laville (Éd.), Variations sur une leçon de mathématiques. Analyses d'une séquence : "L'écriture des grands nombres ». Paris : L'Harmattan.

RICHARD, J.-F. (1990). Les activités mentales. Paris : Colin.

RIPoLL, H. (1985). L'apprentissage du mouvement, in Psychopédagogie des Activités Physiques et Sportives. P. Arnaud \& G. Broyer (Éds), Toulouse : Privat, 291-310.

Sensevy, G., Mercier, A. \& Schubauer-Leoni, M.-L. (2000). Vers un modèle de l'action didactique du professeur à propos de la course à 20, Recherches en Didactique des Mathématiques. 20, 3, 263-304.

THÉPAUT, A. (1992). Culture corporelle et E.P.S. à l'école élémentaire: les paradoxes de l'initiation sportive. Mémoire de Maîtrise sous la direction de J. Hédoux et P. Demunter, UFR des Sciences de l'Éducation et des Pratiques de Formation, Université de Lille III.

THÉPAUT, A. (2002). Échec scolaire et éducation physique et sportive à l'école élémentaire. Etude des interactions maître-élèves dans la construction des savoirs, l'exemple de l'apprentissage de la passe en basket-ball. Thèse de doctorat STAPS, non publiée, Université de Rennes 2, France. 


\section{Zusammenfassung : Fine Studie über den Prozess der Wissensabnahme in Mannschaftssportarten. Aktivität der Schüler und Vertragstyp in der Grundschule (3. Klasse)}

Dieser Artikel untersucht die Wissensabnahme während einer Lerneinheit beim Basketball in der Grundschule. Er analysiert die Schüleraktivitäten aus einer systemtheoretischen Perspektive, d.h. in ihrer Verbindung mit zwei anderen Komponenten des didaktischen Systems, nämlich dem Lehrer und dem Wissen. Die Beobachtungen zeigen, dass die Schüler, sobald sie mit einem vom Lehrer gestellten Problem konfrontiert werden, Lösungen suchen und ausprobieren. Diese Schüler sind in Interaktion mit dem „didaktischen Milieu“. Allerdings werden diese Lösungen, da sie nicht durch den Lehrer aufgenommen, diskutiert und analysiert werden, von den Schülern entweder behalten oder vergessen, je nachdem ob sie erfolgreich oder erfolglos waren. Dies kennzeichnet einen „empiristischen Lernvertrag", der typisch für eine offene Lehrweise zu sein scheint, ein pädagogisches Modell, das oft im Sportunterricht dieser Schulstufe beobachtet wird.

SCHLAGWÖRTER : Wissensabnahme,, didaktischer Vertrag, Sportunterricht, Basketball, Grundschule.

\section{RIAssunto : Uno studio del processo di devoluzione dei saperi negli sport collettivi. Attività degli allievi e tipo di contratto nella scuola elementare (ciclo 3)}

Questo articolo studia il processo di devoluzione del sapere nel corso di un episodio di apprendimento della pallacanestro nella scuola elementare. Analizza l'attività degli allievi in una prospettiva sistemica, cioè nelle sue relazioni con le due altre componenti del sistema didattico: il maestro, il sapere. Le osservazioni realizzate mostrano che gli allievi, confrontati ad un problema posto dal maestro, cercano e sperimentano delle soluzioni. Questi allievi sono ben in interazione con "l'ambiente didattico". Tuttavia, queste soluzioni, in mancanza di essere rilevate, discusse ed analizzate con l'insegnante, sono ritenute o no dagli allievi secondo le loro riuscite o i loro insuccessi. Ciò caratterizza un "contratto d'apprendimento empirista" che appare significativo di una pedagogia della sistemazione dell'ambiente, modello pedagogico frequentemente osservato in EFS a questo livelli di scolarità.

PAROLE ChIAVE : contratto didattico, devoluzione dei saperi, EFS, Pallacanestro, scuola elementare.

\section{Resumen : Estudio de procesos de evolución de saberes en los deportes colectivos. Actividades de los alumnos y tipo de contrato en la escuela primaria (3e ciclo)}

Este artículo estudia los procesos de evolución de saberes en una etapa de aprendizaje en básquetbol en la escuela primaria. El análisis y la actividad de los alumnos desde una perspectiva sistémica, es decir en relación con dos otros componentes del sistema didáctico: el profesor y el saber. Las observaciones realizadas nos muestran que los alumnos, confrontados a un problema presentado por el profesor, buscan y experimenta soluciones. Estos alumnos se interaccionan con el medio didáctico. Cada solución es analizada entre los alumnos y el profesor, estas pueden utilizarse o desecharse en función del fracaso o de la victoria. Esto se caracteriza por un contrato de tipo empírico que aparece de manera significativa en una pedagogía de adaptación al medio, modelo pedagógico frecuentemente observado en educación física deportiva en este nivel escolar.

PALABRAS CLAVES : devolución de saberes, contrato didáctico, educación física deportiva, básquetbol, escuela primaria. 